\title{
Control of Local Mass Transfer in the Separated and Reattaching Flow by a Periodic Forcing
}

\author{
M. Bouterra ${ }^{1}$, Z. Mehrez ${ }^{1}$, A.E. Cafsi ${ }^{1}$, A. Belghith ${ }^{1}$ and P.L. Quere $^{2}$ \\ ${ }^{I}$ Faculté des Sciences de Tunis-Campus Universitaire 1060 Tunis TUNISIE \\ ${ }^{2}$ LIMSI-CNRS Bat. 508 - B.P. 133 - 91403 Orsay Cedex FRANCE \\ $†$ Corresponding Author Email: mourad.bouterra@fst.rnu.tn
}

(Received July 23, 2009; accepted January 24, 2010)

\begin{abstract}
A numerical study based on the Large Eddy Simulation (LES) methodology was made of mass transfer in locally forced turbulent separated and reattaching flow over a backward facing step. The local forcing was given to the flow by a sinusoidally blowing /suction of the fluid into a separated shear layer. The Reynolds number was fixed at 33000 and Schmidt number at 1 . The forcing frequency was varied in the range $0 \leq S t \leq 2$, where St is the Strouhal number of forcing. The obtained results revealed the existence of an optimum forcing frequency value, $S t=0.25$, in terms of the reduced reattachment length. At this frequency the mass transfer is significantly enhanced in the recirculation zone. The influence of the frequency and the amplitude of forcing, in the maximum mass transfer positions and the maximum Sherwood number, are analyzed.
\end{abstract}

Keywords: Backward-facing step, Large eddy simulation, Mass transfer, Periodic forcing.

\section{INTRODUCTION}

Separated and reattaching flows, such as a backwardfacing step, occurs in many engineering problems. Separated flows with reattachment give rise to flow unsteadiness, enhancement of flow mixing, pressure fluctuations and noise. Also, they tend to enhance heat and mass transfer and augment mixing. For this reason the study of control of this type of flow is interesting. Many attempts have been made to control turbulent separated and reattaching flows. Bhattacharjee et al. (1986), Chun et al. (1998), Yoshioka et al. (2001), Rhee et al. (2000), Dejoan et al. (2004), Mehrez et al. (2010), have studied the effects of periodic external forcing on the separated and reattaching flows. They showed that the applied local forcing of the edge step enhances the shear layer growth rate and generates a large scale vortex in the vicinity of the separation side. They also showed the existence of optimal value of forcing frequency in term of reduction of the reattachment length.

In this paper, by a numerical methodology Large Eddy Simulation: LES, we study the effect of a periodic forcing on the dynamic of the flow and mass transfer over a backward-facing step.

\section{Problem DESCRIPTION}

Two-dimensional flow behind a backward-facing step subjected to a sinusoidal forcing is simulated. The computational domain and the coordinates system are represented in Fig. 1. Channel expansion ratio is fixed at $\mathrm{H} / \mathrm{h}=3$ in all the study. The Reynolds number is fixed at 33000. To control the flow, Chun et al. (1998), Yoshioka et al. (2001), experimentally introduced a periodic forcing by horizontal pulsating jet (blowing and suction of the fluid) at the edge of the step. In this work, we have simulated such a forcing by introducing a local velocity $u=A \sin (2 \pi f t$ ) (Fig. 1.), where $A$ and $f$ are respectively the amplitude and the frequency of locally forcing. The mass source " $\mathrm{S}$ " is placed upstream of the step at the concentration $C=1$. At the channel inlet, a fully developed parabolic profile for the velocity is deployed. At the exit, convective boundary conditions for all variables $\left(\frac{\partial u}{\partial x}=\frac{\partial v}{\partial x}=\frac{\partial C}{\partial x}=0\right)$ are set. No-slip conditions are prescribed at the body surfaces $(u=v=0)$. At the upper boundaries, symmetry conditions simulating a frictionless wall are used $\left(\frac{\partial u}{\partial y}=v=\frac{\partial C}{\partial y}=0\right)$. 


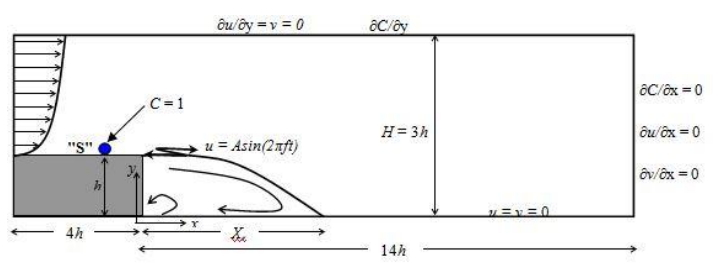

Fig. 1. Geometric configuration of computational domain.

\section{BaSiC Equations}

In this paper, the L.E.S approach is used to solve the Navier-Stokes equations for its principle benefit: simulating only the large scales of turbulence and taking into account the small scales by a mixed subgrid-scale model proposed by Ta Phuoc (1994) and Sagaut (1998) and also used by Leonard (1988).

With reference to Newtonian incompressible fluid of constant physical properties, the equations are written in dimensionless form as:

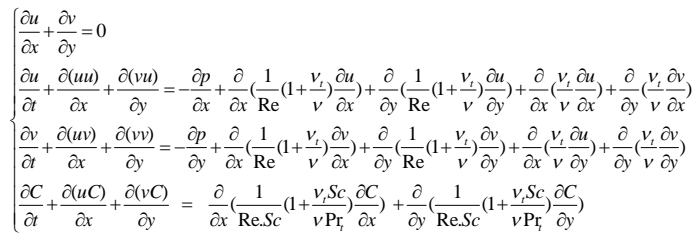

$\mathrm{x}, \mathrm{y}$ are the dimensionless Cartesian coordinates; $u$ and $v$ are respectively the components horizontal and vertical of velocity. The governing dimensionless parameter appearing in the above equations is the Reynolds number $\operatorname{Re}=\frac{U_{o} h}{v}$. When $\mathrm{U}_{\mathrm{o}}$ denote the reference values of streamwise mean velocity and $v$ is the kinematics viscosity. $S c$ is the Shmidt number fixed at $S c=1$.

The numbers of molecular and turbulent Prandtl are fixed at $\operatorname{Pr}=0,7$ and $P r_{t}=1$. The turbulent viscosity is given by $v_{t}=c_{m}|\bar{S}|^{\alpha}\left(q_{c}^{2}\right)^{(1-\alpha) / 2} \Delta^{(1+\alpha)}$. When considering homogeneous isotropic turbulence, and $\alpha=0.5$ (the value retained throughout this work), the theoretical value of the parameter $c_{m}$ is found equal to 0.04 on the basis of an equilibrium assumption between the dissipation and energy-transfer rates. The characteristic length scale is usually chosen to be $\Delta=\sqrt{\Delta x \Delta y}$, where

$\Delta \mathrm{x}$ and $\Delta \mathrm{y}$ are mesh sizes in the $\mathrm{x}$ - and $\mathrm{y}$-directions respectively. The second invariant of the shear stress tensor is given by $|\bar{S}|=\left(\bar{S}_{i j} \bar{S}_{i j}\right)^{1 / 2}$, where $\bar{S}_{i j}=\frac{1}{2}\left(\frac{\partial \bar{u}_{i}}{\partial x_{j}}+\frac{\partial \bar{u}_{j}}{\partial x_{i}}\right)$ is the strain rate tensor of the filtered flow field, and the kinetic energy $q_{c}^{2}$ is obtained by the scale similarity assumption, and by means of a doublefiltering technique: $q_{c}^{2}=\frac{1}{2}\left(\bar{u}_{i}-\hat{\bar{u}}_{i}\right)^{2}$, where $\left(^{\wedge}\right)$ represents a filter with a cut-off length of $2 \Delta$. The explicit filter used here is a local weighted-average $\hat{\hat{u}_{i}}=\frac{1}{4} \bar{u}_{i-1}+\frac{1}{2} \bar{u}_{i}+\frac{1}{4} \bar{u}_{i+1}$.
The mixed subgrid scale model is a self-adapted model because the eddy viscosity vanishes automatically at the wall and in the regions of the flow where all the structures are well resolved.

\section{NUMERICAL METHODOLOGY}

A finite volume method is adapted to the standard staggered grid in which pressure and velocity components are stored at different points. On the other hand, we have used the projection (fractional step) method (Chorin (1968) and Temam (1968)) for decoupling pressure from velocity. The space discretization uses a second order upwind finite difference method by means of a QUICK scheme, proposed by Leonard (1988) for the convective terms and a centered scheme for the diffusive ones.

\section{ReSUlts AND Discussions}

\subsection{Grid and Time-Step Independence}

Grid refinement tests have been performed using three uniform fine grids: $66 \times 34,104 \times 66$ and $130 \times 75$ for $R e=33000$. Results showed that when we pass from the first grid to the second, the reattachment length $X_{r} / h$ without an applied periodic forcing undergoes an increase of $7.1 \%$. When we pass from the second grid to the third, the reattachment length undergoes an increase of only $1.12 \%$. We conclude that the grid of $104 \times 66$ gives a good compromise between precision and calculation time and is sufficient to carry out a numerical study of this flow.

The time-step $\Delta \mathrm{t}$ is conditioned by the CourantFriedrich-Lewy (CFL) criterion. The adjustment of its value is undergone using the optimal $104 \times 66$ grid. Some preliminary runs showed that the dimensionless time interval $t U_{d} / H=400$ is large enough to reach the asymptotic regime considered in the current study. Accordingly, we have done three simulations with $\Delta t$ equal to $8.10^{-3}, 4.10^{-3}$, and $10^{-3}$. Obtained reattachment length values are respectively; $X_{r} / h=6.7,7.2$ (i.e an increase of $6.94 \%$ with respect to the previous value) and 7.28 (i.e an increase of only 1.1\%). Basing on these data, and calculation-time consuming considerations, the time-step value is finally set to $4.10^{-3}$. It's worth to remind here, that for $\Delta t=10^{-3}, 400000$ time steps are necessary to reach the asymptotic regime. This number falls down to 100000 for $\Delta t=4.10^{-3}$.

\subsection{Structure of Non-Forcing Flow}

The iso-stream function lines are represented in the Fig. 2. We can note the existence of a recirculation zone behind the step, where the flow closes against itself to come back toward the step and forms a vortex. This zone is limited by the zone of reattachment, at the end of which the boundary layer redevelops. It is to be noted that under the step in the corner, a second recirculation is generated. The same flow pattern was observed by Scharm et al. (2004) and Bouda et al. (2008). The value of the time-mean reattachment length (indicated by a triangle in the Fig. 2) in the experiment of Chun and Sung (1998) is $X_{r o}=7.4 h$, our simulations 
give a value of $X_{r o}=7.2 \mathrm{~h}$. The agreement between the computation and the experiment is rather good.

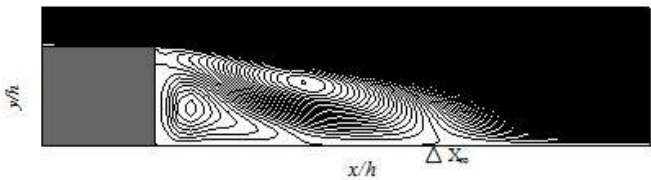

Fig. 2. Distributions of iso-stream function lines. $R e=33000$, without control

In the Fig. 3, the average field of concentration is presented. The most important values of concentration exist upstream of the step. In the downstream the enlargement of concentration zones is observed. These homogeneous areas contribute to a better dispersion mass. These results are in concord with those found in experimental work of Grazzini et al. (1996) and numerical works of Dargent et al. (1996) and Bouterra et al. (2008)

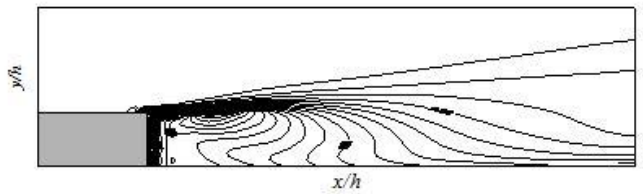

Fig. 3. Distributions of iso-concentration lines. $R e=$ 33000 , without control

\subsection{Influence of the Control on Reattachment Length}

In the Fig. 4 the Iso-stream function lines are plotted at the forcing amplitude $A=0.3 U_{o}$, for different Strouhal number $\mathrm{St}=\mathrm{fh} / \mathrm{U}_{\mathrm{o}}$ based on the forcing frequency $f$, the step height $\mathrm{h}$ and the streamwise velocity $U_{o}$. It is noted the reattachment length (mentioned in the figure by the triangle) and the size of recirculation zone reduces by application of local forcing. The optimum value of forcing frequency in term of reduction reattachment length is $S t=0.25$. This is in agreement with the experiment works of Chun et al. (1998) who found a value of Strouhal number $S t=0.2$. It is known that the shear layer above the recirculation zone is the seat of formation of coherent vortical structures. These vortices that are convecting downstream are responsible for the entrainment out of the recirculation zone. By stimulating their growth through applying a local forcing, the increased shear layer growth rate leads to a significant reduction of the recirculation zone size.

\subsection{Influence of the Control on Mass Transfer}

\subsubsection{Influence of the forcing frequency}

In this section the amplitude is fixed at $A=0.3 U_{o}$. Fig. 5 shows the local Sherwood number $S h=-\mathrm{h}(\partial \mathrm{C} / \partial \mathrm{y})_{\mathrm{w}}(C$ is the concentration and $\mathrm{w}$ indicates the bottom wall downstream of the step) profiles along the bottom walls for various Strouhal number values. All the local Sherwood number profiles show the same general features. There is a low mass transfer in the recirculation zone followed by a steep rise to the maximum near the reattachment point.

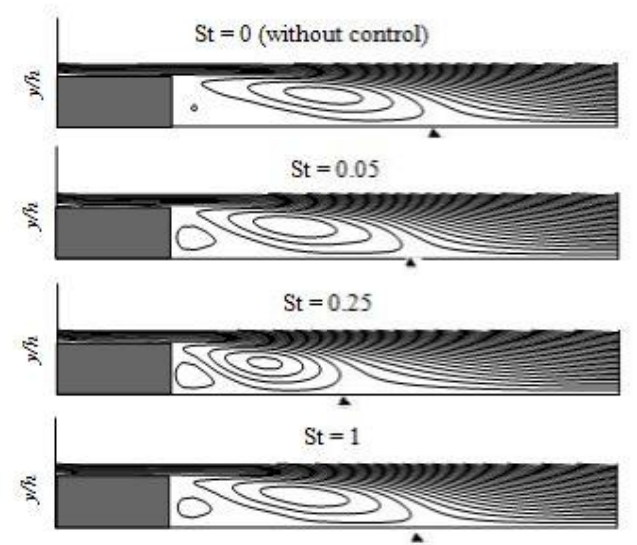

Fig.4. Iso-stream function lines for different Strouhal number $S t$.

Then, it gradually decreases in the redevelopment zone. The location of maximum mass transfer abscissa $X_{m}$ is around of reattachment for different cases. This result is in good agreement with the experiment of Hwang et al. (1998). The local forcing increases the mass transfer in the recirculation zone and shifts the point of the maximum transfer towards the step. This last observation is confirmed by the effect of forcing frequency on $X_{m} / X_{m o}\left(X_{m o}\right.$ is the abscissa of the maximum mass transfer in the non-forcing case) (Fig. 6). In fact, $X_{m}$ decreases by the application of local forcing. The minimum is obtained at the optimum frequency $(S t=0.25)$. The shear layer in the vicinity of the sharp separation edge is modified by the local forcing. This modification gives rise to a large increase in the entrainment close to the separation edge, which increases the shear layer growth rate. As a consequence, the size of recirculation decreases and the mass transfer, between the flow from the inlet and the wall, enhances.

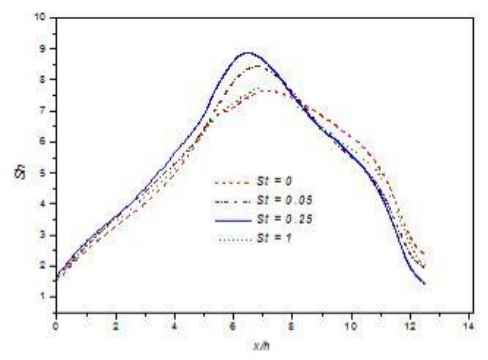

Fig. 5. Local Sherwood number profiles for four Strouhal number values. $A=0.3 U_{o}$.

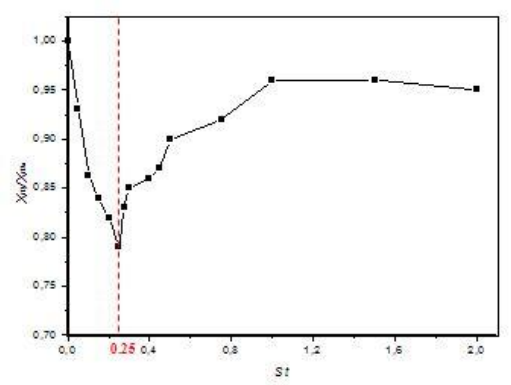

Fig. 6. Normalized maximum mass transfer abscissa $X_{m} / X_{m o}$ against $S t . A=0.3 U_{o}$ 


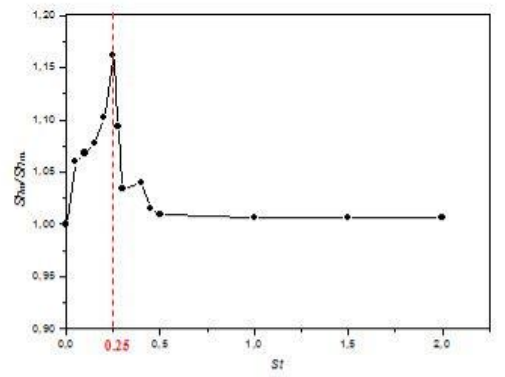

Fig.7. Normalized maximum local Sherwood number $S h_{m} / S h_{m o}$ against St. $A=0.3 U_{o}$.

The normalized maximum local Sherwood number $S h_{m} / S h_{m o}\left(S h_{m o}\right.$ is the value at non-forcing flow case) is plotted in Fig. 7 as a function of the Strouhal number $S t$. At the optimum frequency $S t=0.25$, it is seen that the maximum mass transfer is most enhanced (this is confirmed by the observation of the Fig. 5). This suggests that the mass transfer is affected by large-scale vortical structure introduced by forcing in a flow field; i.e, the large-scale structure plays an important role in the enhancement of mass transfer.

\subsubsection{Influence of the Forcing Amplitude}

In this section, the Strouhal number is fixed at optimum value $S t=0.25$, where the increase of mass transfer rate in the recirculation zone is maximum. In Fig. 8 the dependence of the local Sherwood number profile of forcing amplitude is shown. The increase of forcing amplitude: i) enhances the mass transfer in the recirculation zone, ii) shifts the point of the maximum mass transfer towards the step, and, iii) enhances the $\mathrm{Sh}_{\mathrm{m}}$ value. This is explained by the intensification of the large-scale vortical structure by increasing the forcing amplitude. In Fig. 9 the variation of the $X_{m} / X_{m o}$ against the forcing amplitude is plotted. As can be seen, $X_{m}$ continuously decreases with increasing $A / U_{o}$ until $A$ $=U_{o}$. Starting from this point, no significant effects of the variation of amplitude are observed. The maximum reduction of $X_{m}$, compared to non-forcing case, is $20 \%$. This is accompanied by the increase of the maximum Sherwood number $S h_{m}$ until a limiting value, at which $30 \%$ of augmentation (compared to non-forcing case) is observed (see Fig. 10.). It is concluded that the enhancement of the mass transfer rate in the recirculation zone, observed at the optimum forcing frequency, can be risen or reduced following the improvement or attenuation of the vortical intensity by acting on the forcing amplitude at the optimum frequency.

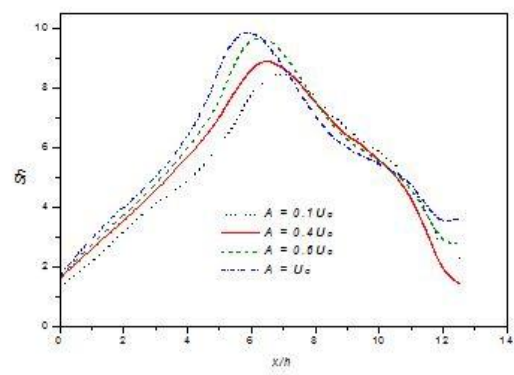

Fig. 8. Local Sherwood number profiles for different forcing amplitude values at $S t=0.25$.

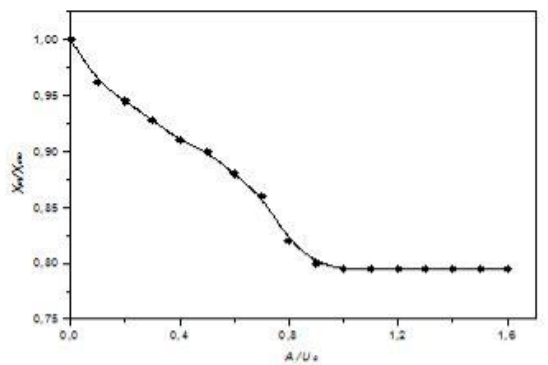

Fig. 9. Normalized maximum mass transfer abscissa $X_{m} / X_{m o}$ against $A / U_{o}$ at $S t=0.25$

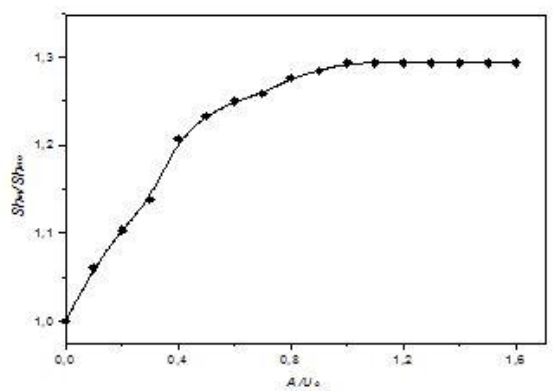

Fig. 10. Normalized local Sherwood number $S h_{m} / S h_{m o}$ against $A / U_{o}$ at $S t=0.25$.

In summary, the overall convective mass transfer rate is found to increase when the local forcing is imposed. It is worth pointing out that this finding is analogous to the case of heat transfer (Mehrez et al. (2009))

\section{Conclusion}

In this study, mass transfer and fluid dynamics of the separated and reattaching flow past a backward- facing step have been simulated using Large Eddy Simulation methodology. The flow is subjected to a sinusoidal forcing. It has been found that the applied of local forcing at the optimal Strouhal number $S t=0.25$ reduces considerably the reattachment length. At this frequency value a considerable increase in the mass transfer in the recirculation zone, along with a large reduction of the maximum mass transfer abscissa are observed. By increasing the forcing amplitude, the mass transfer in the recirculation zone enhances. The maximum Sherwood number rise is $30 \%$ and the maximum decrease of the abscissa of maximum mass transfer is $20 \%$ compared to non-forcing case. The found results show the possibility of control of mass transfer in the separated and reattaching flows.

\section{REFERENCES}

Bhattacharjee, S., B. Scheelke and T.R. Troutt (1986). Modification of vortex interaction in a reattaching seperated flow. AIAA 24, 623-629.

Bouda, N., N. Schiestel, M. Amielh, C. Rey and T. Benabid (2008). Experimental approach and numerical prediction of turbulent wall jet over a backward facing step. Int. J. of Heat and Fluid Flow 29, 927-944. 
Bouterra, M., A. El Cafsi, A. Belghith and P. Le Quéré (2008). Etude numérique de la dispersion d'un polluant autour d'un obstacle soumis à un écoulement turbulent perturbé. Revue des Energies Renouvelables 11, 485-491.

Chun, K.B. and H.J. Sung (1998). Visualization of a locally- forced separated flow over a backwardfacing Step. Exp.Fluids 25, 417-426.

Chorin, A. (1968). Numerical simulation of the Navier-Stokes equations. J. Math. Comput. 22, 745-762.

Dargent, C., F. Grazzini, J. George and D. Dartus (1996). Numerical Simulation of a pollutant dispersion around an obstacle. Proceedings of the International Conference, Air Pollution, 257266.

Dejoan, A. and M.A. Leschziner (2004). Large eddy simulation of periodically perturbed separated flow over a backward-facing step. Int. J. Heat Fluid Flow 25, 581-592.

Grazzini, F., C. Dargent, J. George and D. Dartus (1996). An experimental study of several parameters influencing the phenomenon of vortex shedding behind a square cylinder. In Proceedings of the International Conference, Air Pollution, 297-304.

Hwang, K.S., H.J. Sung and J.M. Hyun (1998). Flow and mass transfer measurements for a flat plate of finite thickness in pulsating flow. Int. $J$ of Heat and Mass Transfer 41, 2827-2836.

Leonard, B.P. (1988). Simple high accuracy resolution program for convection modelling of discontinuities. Int. J. for Num. Methods in Fluids 8, 1291-1318.

Mehrez, Z., M. Bouterra, A. El Cafsi, A. Belghith and P. Le Quéré (2009). The influence of the periodic disturbance on the local heat transfer in separated and reattached flow. Journal of Heat and Mass Transfer. 46(1), 107-112.

Mehrez, Z., M. Bouterra, A. El Cafsi, A. Belghith and P. Le Quéré (2010). Simulation of the Periodically Perturbed Separated and Reattaching Flow over a Backward-Facing Step. Journal of Applied Fluid Mechanics 3(2), 1-8.

Rhee, G.H. and H.J. Sung (2000). Turbulent Numerical prediction of locally forced separated and reattaching flow. Fluid Dynamics Research 26, 421-436.

Sagaut, P. (1998). Introduction à la Simulation des Grandes échelles Pour les écoulements de Fluide Incompressible. Book. Math. Springer-Verlag, Collection Mathématiques et applications 30, 282 pages.

Scharm, C., P. Rambaud and M.L. Riethmuller (2004). Wavelet based eddy structure deduction from a backward facing step flow investigation using a particle image velocimetry. Exp. Fluids 36, 233245 .

Ta Phuoc, L. (1994). Modèles de sous maille appliqués aux écoulements instationnaires décolles, DGA/DRET. Journée Thématique DRET Aérodynamique Instationnaire Turbulent, Aspects Numériques et Expérimentaux, France.

Temam, R. (1968). Une méthode d'approximation de la solution des équations de Navier-Stokes. Bull. Soc. Math. France 98, 115-152.

Yoshioka, S., S. Obi. And S. Masuda (2001). Organized vortex motion in periodically perturbed turbulent flow over a backward-facing step. Int. J. Heat Fluid Flow 22, 301-307. 